\title{
Previsión de la temperatura superficial del mar frente a la costa perua- na mediante un modelo autorregresivo integrado de media móvil
}

\section{Forecast of sea surface temperature off the Peruvian coast using an autoregressive integrated moving average model}

\author{
Carlos Quispe ${ }^{1}$ y Sara Purca ${ }^{1}$
}

\begin{abstract}
${ }^{1}$ Centro de Investigaciones en Modelado Oceanográfico y Biológico Pesquero (CIMOBP), Instituto del Mar del Perú (IMARPE), Apdo. 22, Callao, Perú

Email Carlos Quispe: cquispe@imarpe.gob.pe
\end{abstract}

Presentado: $\quad 05 / 01 / 2006$ Aceptado: $\quad 12 / 12 / 2006$

\section{Resumen}

El evento EI Niño conecta globalmente el clima, los ecosistemas y las actividades socioeconómicas. Desde 1980 se ha intentado predecir este evento, pero hasta la fecha los modelos estadísticos y dinámicos aún son insuficientes. Por tal motivo, el objetivo del presente trabajo fue explorar mediante un modelo autoregresivo integrado de media móvil el efecto de El Niño en la temperatura superficial del mar (TSM) frente a la costa peruana. El trabajo comprendió 5 etapas: identificación, estimación, verificación diagnóstica, previsión y validación. Se usaron las funciones de autocorrelación simple y parcial (FAC y FACP) para identificar y reformular los órdenes de parámetros en los modelos, así también los criterios de información de Akaike (AIC) y de Schwarz (SC) para la selección de modelos en la verificación diagnostica. Entre los principales resultados se propuso modelos ARIMA $(12,0,11)$ que simularon condiciones mensuales similares a las observadas en el litoral peruano: condiciones frías a fines del 2004, y condiciones neutrales a inicios del 2005.

Palabras clave: ENOS, modelo ARIMA, temperatura superficial del mar, series temporales, Perú.

\section{Abstract}

El Niño connects globally climate, ecosystems and socio-economic activities. Since 1980 this event has been tried to be predicted, but until now the statistical and dynamical models are insufficient. Thus, the objective of the present work was to explore using an autoregressive moving average model the effect of El Niño over the sea surface temperature (TSM) off the Peruvian coast. The work involved 5 stages: identification, estimation, diagnostic checking, forecasting and validation. Simple and partial autocorrelation functions (FAC and FACP) were used to identify and reformulate the orders of the model parameters, as well as Akaike information criterium (AIC) and Schwarz criterium (SC) for the selection of the best models during the diagnostic checking. Among the main results the models $\operatorname{ARIMA}(12,0,11)$ were proposed, which simulated monthly conditions in agreement with the observed conditions off the Peruvian coast: cold conditions at the end of 2004, and neutral conditions at the beginning of 2005.

Keywords: ENSO, ARIMA model, sea surface temperature, time series, Peru.

\section{Introducción}

La temperatura superficial del mar (TSM) es una de las variables físicas que mejor indica la variación temporal del ciclo El Niño y la Oscilación del Sur (ENOS), por lo cual es ampliamente usada para estudiar la variabilidad a diferentes escalas de tiempo en el Océano Pacífico (Purca et al. 2005). Los efectos de los extremos opuestos del ciclo ENOS (El Niño y la Niña) producen cambios severos en el Ecosistema de la Corriente de Humboldt (Purca et al. 2005) y cambios climáticos asociados a diferentes desastres naturales (McPhaden, 2001), lo cual se traduce en fuertes impactos en las actividades socioeconómicas de la región occidental del América del Sur. Actualmente, el índice operacional "Oceanic Niño Index" (ONI) evalúa el estado del ciclo ENOS (NOAA Magazine, 2003), este índice está en función de las anomalías de temperatura superficial del mar y está definido para una región crítica del Pacífico Central Ecuatorial (región Niño 3.4; $120^{\circ} \mathrm{W}-170^{\circ} \mathrm{W}, 5^{\circ} \mathrm{N}-5^{\circ} \mathrm{S}$ ). La región Niño 3.4, por su ubicación geográfica delimitada, es declarada área de desarrollo del evento El Nińo y La Niña, y el ONI refleja esta variación en el tiempo. En el Perú, con otro escenario geográfico, el clima ha estado influido por procesos físicos, atmosféricos y oceánicos locales muy diferentes a la región Nińo 3.4, por lo que se requiere usar otro índice de variación temporal, que represente los patrones locales, así como también los efectos del ciclo ENOS. El Indice de Oscilación Peruano (IOP), propuesto por Purca (2005), representa la variabilidad espacial conjunta de las anomalías de temperatura superficial del mar (ATSM) frente a la costa peruana.

El presente trabajo no pretende simular el desarrollo del ciclo ENOS en el Pacífico Central, sino más bien simular los efectos del ciclo ENOS sobre el mar peruano, tomando en cuenta sólo procesos costeros.

Debido a la base estocástica de los modelos ARIMA, y a la complejidad de los mecanismos causales de ENOS, se realizarán previsiones del IOP sin asumir un modelo conceptual a priori. El modelado estadístico con el enfoque de los procesos estocásticos, presenta una variedad de funciones lineales que han sido ampliamente usadas para resolver problemas de previsión con datos registrados en series de tiempo. El modelo autorregresivo integrado de media móvil (ARIMA), desarrollado por Box y Jenkins (Box et al. 1994), permite simular el comportamiento de una serie temporal mediante un esquema simplificado de observaciones y choques aleatorios pasados, sin la necesidad de tener en cuenta una concepción a priori del modelo. Existen pocos trabajos sobre modelado y predicción de las variables indicadoras de ENOS y sus efectos frente a la costa peruana, como el trabajo de Woodman (2005), quien establece una relación empírica entre la TSM frente a la costa norte del Perú y las precipitaciones en la ciudad de Piura. 
El objetivo del presente trabajo fue ajustar un modelo estadístico autorregresivo y de media móvil para obtener previsiones a corto plazo del Índice de Oscilación Peruano (IOP), con el fin de evaluar los efectos de El Niño frente a la costa peruana.

\section{Materiales y metodos \\ Datos}

El área de estudio comprende la franja costera del Perú. Los datos mensuales de temperatura superficial del mar (TSM) durante el periodo 1950-2004, fueron obtenidos de las estaciones costeras fijas del Instituto del Mar del Perú (IMARPE). Las estaciones usadas fueron: Chicama $\left(07^{\circ} 42^{\prime} 00^{\prime \prime} \mathrm{S}, 79^{\circ} 27^{\prime} 00^{\prime \prime} \mathrm{W}\right)$, Chimbote $\left(09^{\circ} 04^{\prime} 00^{\prime \prime}\right.$ S, $\left.78^{\circ} 36^{\prime} 00^{\prime \prime} \mathrm{W}\right)$, Huacho $\left(10^{\circ} 16^{\prime} 00^{\prime \prime}\right.$ S, $\left.78^{\circ} 36^{\prime} 00^{\prime \prime} \mathrm{W}\right)$, Callao $\left(12^{\circ} 13^{\prime} 00^{\prime \prime} \mathrm{S}, 77^{\circ} 09^{\prime} 00^{\prime \prime} \mathrm{W}\right)$, Ilo (18 $01^{\circ} 00^{\prime \prime}$ S, $\left.75^{\circ} 09^{\prime} 00^{\prime \prime} \mathrm{W}\right)$.

Se cálculo del Indice de Oscilación Peruano (IOP) usando la metodología de Purca et al. (2005). Se clasificaron las previsiones del IOP en condiciones mensuales (fría, neutra, cálida), a partir de terciles (Enfield et al., 2003) del promedio trimestral del IOP,

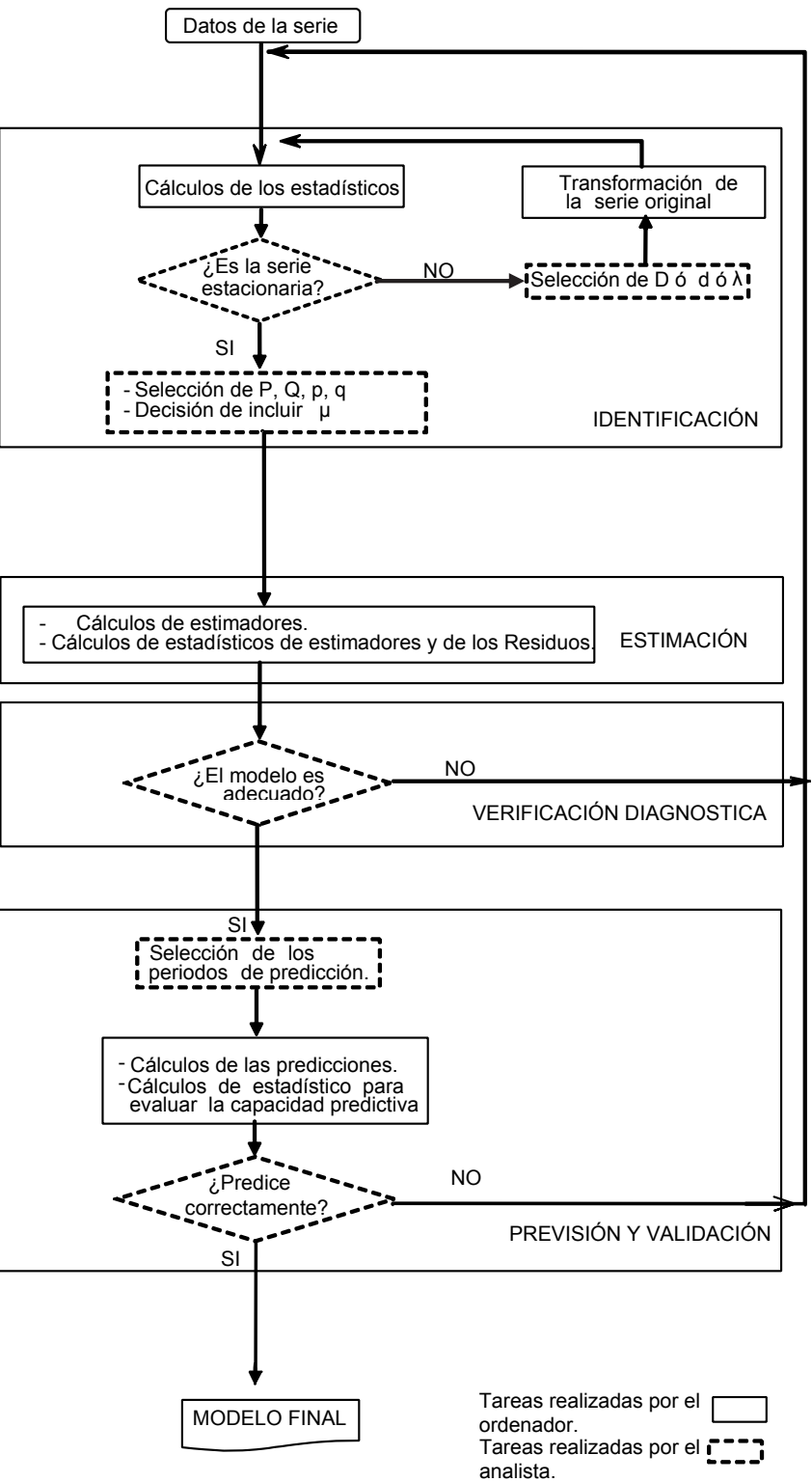

Figura 1. Flujograma con las etapas de desarrollo de modelos estadísticos (modificado de Uriel y Peiró, 2000; Box y Jenkins, 1994; Chatfield 1996).

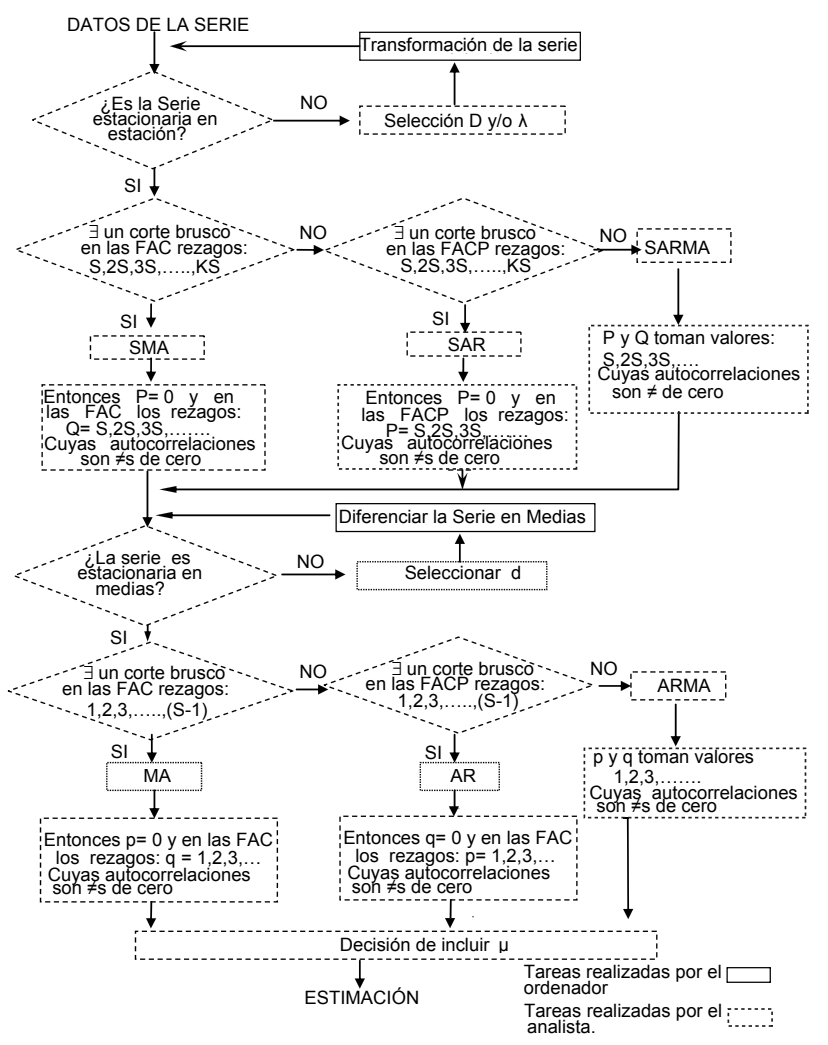

Figura 2. Flujograma detallado de la etapa de identificación.

de manera análoga a la definición operacional de El Niño y La Niña de la NOAA (2003).

\section{Modelos}

El procedimiento de previsión se basó en modelos autorregresivos integrados de media móvil (ARIMA), también conocidos como modelos de Box y Jenkins (Box et al., 1994). El proceso de previsión tiene 5 etapas principales (Uriel \& Peiró, 2000; Chatfield, 1996): identificación, estimación, verificación diagnóstica, previsión y validación (Fig. 1).

La forma general de un modelo autorregresivo integrado de media móvil, ARIMA(p,d,q), es:

$$
\begin{aligned}
& \phi_{p}(\mathrm{~B}) W_{t}=\theta_{q}(\mathrm{~B}) \varepsilon_{t} \\
& \text { donde: }
\end{aligned}
$$

$\mathrm{B}$ es operador de retardos que aplicado a una variable $\mathrm{W}$, la retarda $\alpha$ períodos $\left(\mathrm{B}^{\alpha} \mathrm{W}_{\mathrm{t}}=\mathrm{W}_{\mathrm{t}-\alpha}\right), \phi_{p} \mathrm{y} \theta_{q}$ son polinomios de orden p y q respectivamente, $\varepsilon_{\mathrm{t}}$ es un proceso puramente aleatorio.

$W_{t}=\Delta^{d} \mathrm{Y}_{t}^{(\lambda)}$

donde:

$\mathrm{Y}_{t}$ es la serie original, $\mathrm{d}$ es el orden de diferenciación necesario para alcanzar la estacionariedad y puede tomar valores: $0,1,2$, $\mathrm{W}_{t}$ es la serie estacionaria obtenida por diferenciación de la serie original, $\lambda$ es la potencia de transformación de Box-Cox, p es el orden del término autorregresivo, $\mathrm{q}$ es el orden del término de media móvil.

La etapa de identificación (Fig. 2) comprendió primero, la evaluación de la necesidad de una transformación de Box y Cox para estabilizar la varianza (Chatfield, 1996), y luego el análisis de las funciones de autocorrelación simple (FAC) y parcial (FACP) de los datos originales y transformados para determinar estruc- 


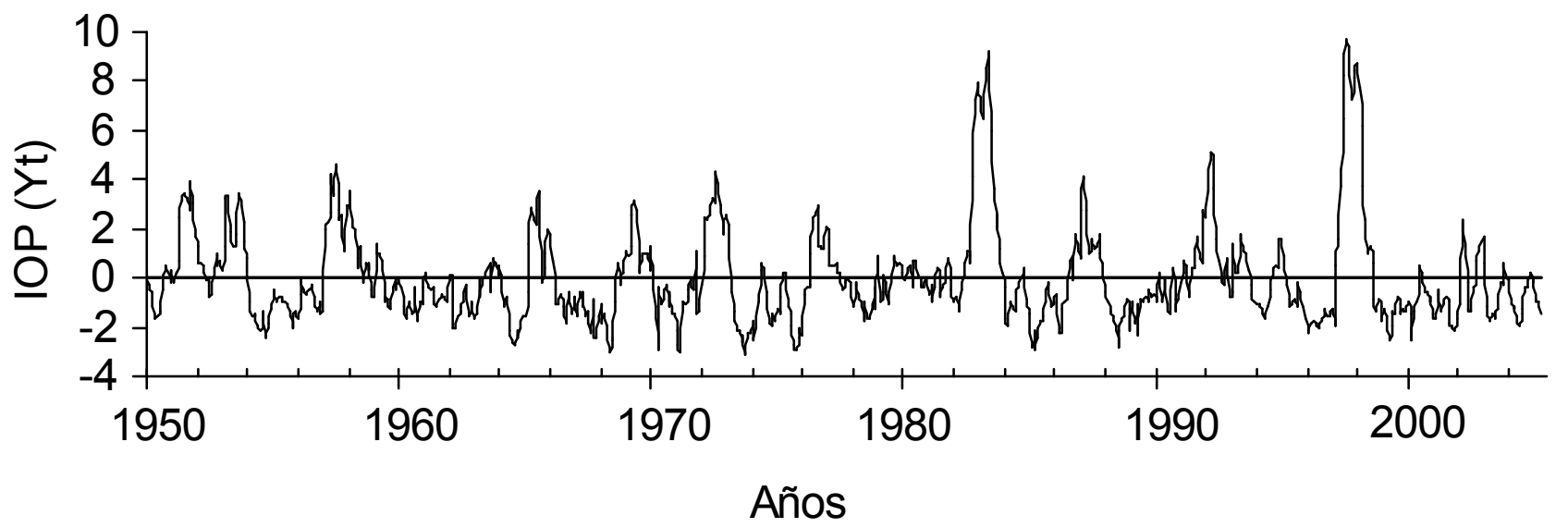

Figura 3. Variación temporal de la serie $Y_{t}$, del Índice de Oscilación Peruano (IOP) desde enero 1950 hasta Abril 2005.

turas tentativas de los órdenes de los parámetros $(\mathrm{p}, \mathrm{d}, \mathrm{q})$.

La etapa de estimación de los coeficientes del modelo ARIMA, se realizó con el software Eviews 3.1 (Quantitative Micro Software 1998), que emplea técnicas de regresión no lineal, y son asintóticamente equivalentes a las estimaciones por máxima verosimilitud.

En la etapa de verificación diagnóstica se evaluó la significancia estadística de los coeficientes estimados, y se examina los residuales para comprobar si los residuos son ruido blanco mediante el estadístico de Ljung-Box. Se realizó un examen del FAC y FACP de los residuos, verificando los supuestos de que:

$\mathrm{E}\left(\boldsymbol{\varepsilon}_{t}\right)=0, \forall \mathrm{t} \quad$ la media es constante e igual a cero

$\operatorname{Var}\left(\boldsymbol{\varepsilon}_{t}\right)=\sigma^{2}, \forall \mathrm{t}$ la varianza es constante

$\mathrm{E}\left(\boldsymbol{\varepsilon}_{t} \cdot \boldsymbol{\varepsilon}_{t}\right)=0, \forall \mathrm{t} \neq \mathrm{t}^{\prime}$ no hay autocorrelación del término estocástico.

Los criterios de selección del modelo más adecuado fueron: el criterio de información de Akaike (AIC) y el criterio de Schwarz (SC), basados en la varianza de los residuos, y cuyos valores comparan modelos de diferentes números de parámetros (Goodrich, 1989). Así el mejor modelo fue aquel con valores menores de los estadísticos AIC y SC.

La etapa de validación se realizó de 3 maneras: i) una validación retrospectiva, simulando el efecto de episodios El Niño históricos; ii) una validación cuantitativa midiendo las bondades

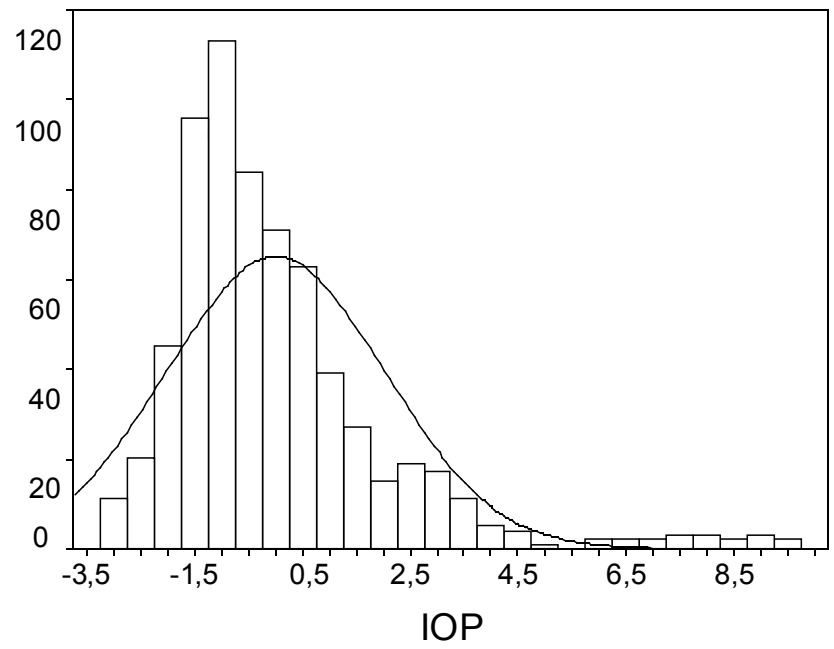

Figura 4. Histograma del Índice de Oscilación Peruano (IOP). de la previsión tomando en cuenta el periodo de julio 2004 al abril 2005, utilizando medidas resúmenes de errores de previsión, como la raíz cuadrada del error medio cuadrático (REMC), la desviación absoluta de la media (DAM), y el porcentaje de error medio absoluto (PEMA); iii) una validación cualitativa comparando las condiciones mensuales simuladas y observadas.

\section{Resultados y discusion \\ Identificación}

Debido al tratamiento de estandarización en función de la varianza, el IOP no presentó una tendencia (Fig. 3), lo cual implica que puede ser una serie de tiempo estacionaria en media. Tomando en cuenta las medidas de localización, de escalas y el histograma (Fig. 4), la distribución de frecuencias del IOP tuvo una forma asimétrica positiva, la distribución de la variación se concentra en las anomalías negativas o períodos fríos debido al sistema de surgencia peruano, lo que hace que predomine aguas frías (Purca 2005). El gráfico de dispersión vs. nivel (Fig. 5) mostró una variabilidad proporcional al nivel de TSM, lo cual implica que la serie es no estacionaria en varianza, entonces es necesario realizar una trasformación logarítmica (Chatfield, 1996).

La nueva serie de tiempo transformada fue $\mathrm{w}_{\mathrm{t}}=\log _{\mathrm{e}}(\mathrm{IOP}+4)$. La función de autocorrelación simple (FAC) y la función de

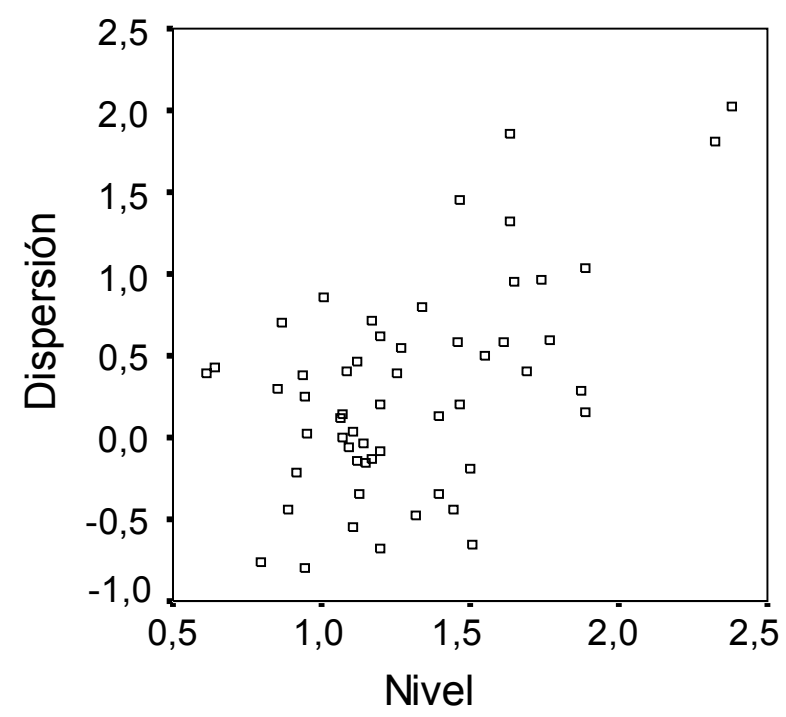

Figura 5. Diagrama dispersión-nivel del IOP. 


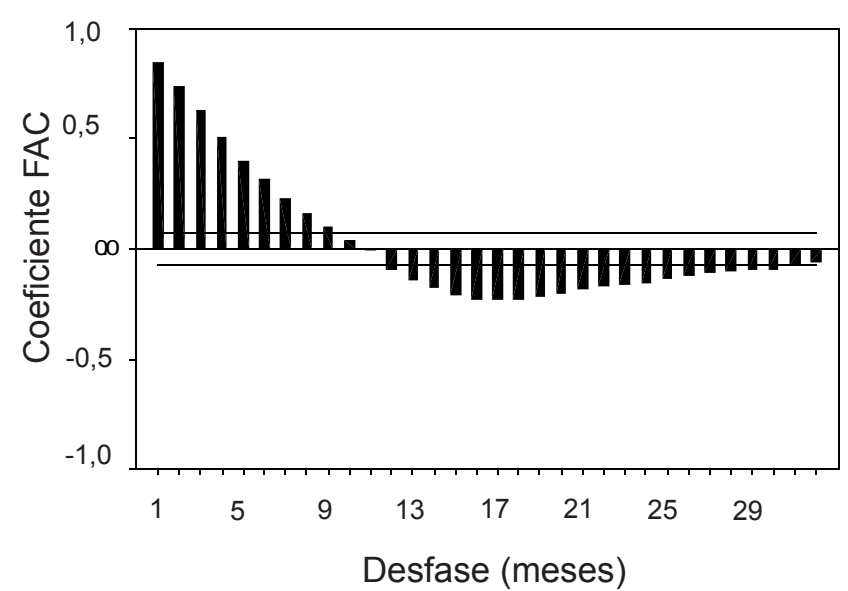

Figura 6. FAC estimada de la serie transformada con logaritmo.

autocorrelación parcial (FACP) de la serie $\left\{\mathrm{w}_{\mathrm{t}}\right\}$ (Figs. 6 y 7) permitieron identificar algunos órdenes de los parámetros (p,d,q), para los modelos de la serie sin diferenciar $(\mathrm{d}=0)$. La FACP mostró un corte brusco en el primer desfase lo que podría sugerir un término autorregresivo de orden 1. La FAC indicó un proceso de memoria larga decreciente.

De esta manera, a partir de las FAC y FACP (Figs. 6 y 7) se identificaron los siguientes modelos potenciales sin diferenciación, asumiendo que la serie es estacionaria en media:

Modelo 1: ARIMA(12,0,0)

$w_{t}=\delta+\phi_{1} w_{t-1}+\phi_{12} w_{t-12}+\varepsilon_{t}$

Modelo 2: $\operatorname{ARIMA}(12,0,1)$

$w_{t}=\delta+\phi_{1} w_{t-1}+\phi_{12} w_{t-12}+\varepsilon_{t}-\theta_{1} \varepsilon_{t-1}$

Modelo 3: $\operatorname{ARIMA}(12,0,12)$

$w_{t}=\delta+\phi_{1} w_{t-1}+\phi_{12} w_{t-12}+\varepsilon_{t}-\theta_{12} \varepsilon_{t-12}$

Modelo 4: ARIMA(12,0,12)

$$
w_{t}=\delta+\phi_{1} w_{t-1}+\phi_{12} w_{t-12}+\varepsilon_{t}-\theta_{1} \varepsilon_{t-1}-\theta_{12} \varepsilon_{t-12}
$$

\section{Estimación}

Se obtuvieron los estimadores de los coeficientes para los diferentes modelos anteriores, y se construyó una tabla de selección del modelo (Tabla 1) con el mejor ajuste, según los criterios de información de Akaike (AIC) y de Schwarz (SC). El mejor modelo debe presentar los menores valores de los estadísticos (AIC

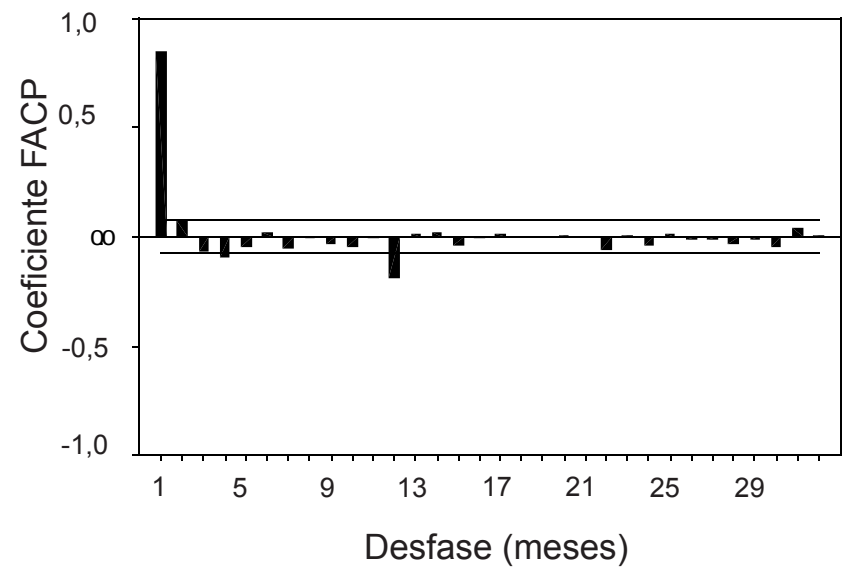

Figura 7. FACP estimada de la serie transformada con logaritmo.
Tabla 1. Criterios de selección del mejor modelo aplicado a la serie con transformación logarítmica.

\begin{tabular}{lccc}
\hline Criterios: & AIC & SC & SSE \\
\hline Modelo 1 & -0.069 & $\mathbf{- 0 . 0 4 8}$ & 34.767 \\
Modelo 2 & $\mathbf{- 0 . 0 7 2}$ & -0.044 & 34.542 \\
Modelo 3 & -0.068 & -0.040 & 34.688 \\
Modelo 4 & -0.071 & -0.036 & $\mathbf{3 4 . 4 6 9}$ \\
\hline
\end{tabular}

y SC), pero hay casos donde ambos criterios no concordaron, principalmente debido a que el criterio de Schwarz (SC) supone una mayor penalización a la introducción de nuevos parámetros (Uriel \& Peiró, 2000), por lo que el SC no seleccionará modelos con mayor número de parámetros que el AIC (parsimonia).

Tomando en cuenta el criterio de Schwarz (SC), el mejor de los modelos sin diferenciación fue el Modelo 1.

En vista de que la FAC de la serie sin diferenciar (Fig. 6) no decayó rápidamente, y mantuvo los coeficientes superiores al desfase 6 estadísticamente significantes, se aplicó una diferencia $(\mathrm{d}=1)$ a la serie de tiempo transformada logarítmicamente, y se identificaron las órdenes ( $\mathrm{p}, 1, \mathrm{q})$ de las FAC y FACP (Figs. 8 y 9), las mismas que mostraron estacionariedad severa (Fig. 9), sugiriendo un término autorregresivo de 1 er orden $(\mathrm{p}=1)$.

De esta manera, a partir de las FAC y FACP (Figs. 8 y 9) se identificaron los siguientes modelos potenciales con diferenciación, dejando algunas alternativas para las medias móviles:

Modelo 5: ARIMA $(1,1,0)$

$$
w_{t}=\left(1+\phi_{1}\right) w_{t-1}-\phi_{1} w_{t-2}+\varepsilon_{t}
$$

Modelo 6: $\operatorname{ARIMA}(1,1,1)$

$$
w_{t}=\left(1+\phi_{1}\right) w_{t-1}-\phi_{1} w_{t-2}+\varepsilon_{t}-\theta_{1} \varepsilon_{t-1}
$$

Modelo 7: $\operatorname{ARIMA}(1,1,11)$

$$
w_{t}=\left(1+\phi_{1}\right) w_{t-1}-\phi_{1} w_{t-2}+\varepsilon_{t}-\theta_{1} \varepsilon_{t-1}-\theta_{11} \varepsilon_{t-11}
$$

Según los estadísticos de AIC y SC, el mejor de los modelos con diferenciación fue el Modelo 7 (Tabla 2).

\section{Verificación diagnóstica}

El análisis residual (FAC y FACP de los residuos) del Modelo 1 (Figs. 10 y 11), indicó que en el desfase 11 hay información relevante, y es necesario reformular este modelo asignando una media móvil de orden 11 al Modelo 1, quedando de la siguiente forma:

Modelo 8 : ARIMA(12,0,11)

$$
w_{t}=\delta+\phi_{1} w_{t-1}+\phi_{12} w_{t-12}+\varepsilon_{t}-\theta_{11} \varepsilon_{t-11}
$$

$\mathrm{Al}$ ajustar el modelo reformulado a la serie de tiempo se obtuvieron estimados de los coeficientes que resultaron ser todos estadísticamente significantes, según pruebas individuales " $\mathrm{t}$ de student” que contrastan la significancía estadística de cada

Tabla 2. Criterios de selección del mejor modelo aplicado a la serie con transformación logarítmica y diferenciación $(\mathrm{d}=1)$.

\begin{tabular}{cccc}
\hline Criterios: & AIC & SC & SSE \\
\hline Modelo 5 & 0.001 & 0.008 & 38.084 \\
Modelo 6 & 0.004 & 0.018 & 38.084 \\
Modelo 7 & $\mathbf{- 0 . 0 1 3}$ & $\mathbf{0 . 0 0 8}$ & $\mathbf{3 7 . 3 4 0}$ \\
\hline
\end{tabular}




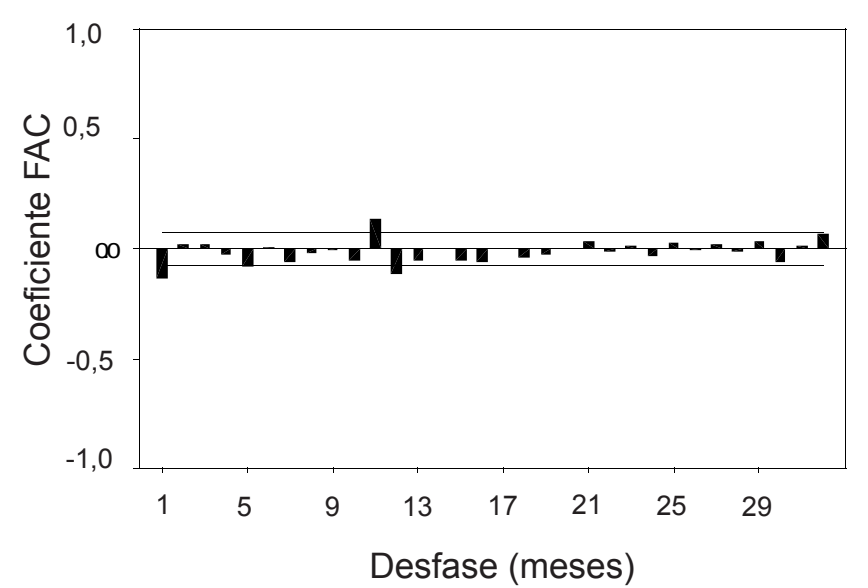

Figura 8. FAC estimada de la serie transformada con logaritmo y diferenciada con desfase $(d=1)$.

Tabla 3. Verificación diagnóstica de los estimados de los coeficientes del Modelo 8.

\begin{tabular}{ccccc}
\hline Parámetro & Estimado & $\begin{array}{c}\text { Error } \\
\text { estándar }\end{array}$ & $\mathrm{t}$ & Prob. \\
\hline$\hat{\delta}$ & 1,283665 & 0,044 & 29,28812 & 0,000 \\
$\hat{\phi}_{1}$ & 0,848174 & 0,020 & 41,73981 & 0,000 \\
$\hat{\phi}_{12}$ & $-0,084220$ & 0,020 & $-4,163983$ & 0,000 \\
$\hat{\theta}_{11}$ & 0,134501 & 0,039 & 3,406862 & 0,000 \\
\hline
\end{tabular}

parámetro estimado (Tabla 3). De esta manera se logró reducir los estadísticos AIC = -0,0824 y SC = -0,0546.

El supuesto de ruido blanco en los residuos se contrastó con la prueba de Ljung-Box usando el estadístico Q-Stat. Para un desfase de 20 el estadístico Q-Stat fue de 16,225 con un valor $\mathrm{p}=$ 0,508; mayor al nivel de significancia de $\alpha=0,05$ establecido, $y$ se concluyó que los residuos no estaban autocorrelacionados, es decir son ruido blanco. En general la verificación diagnóstica no reveló ninguna falta de adecuación del modelo 8 obtenido.

Por otro lado, el análisis residual (FAC y FACP de los residuos) del Modelo 7, indicó que no era necesario reformular este modelo.

\section{Validación}

Para la validación retrospectiva se usaron modelos ARIMA, para simular las condiciones cálidas del IOP en los ańos 1972,

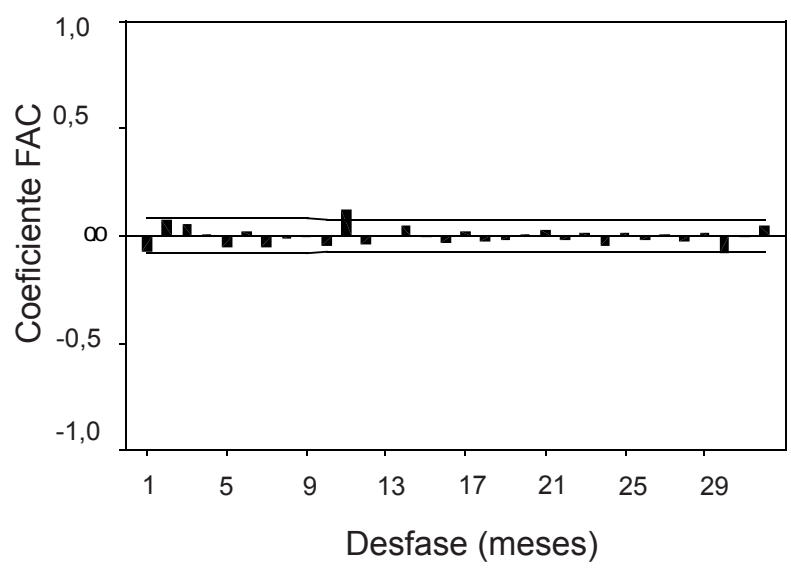

Figura 10. FAC estimada de los residuos del Modelo 1.

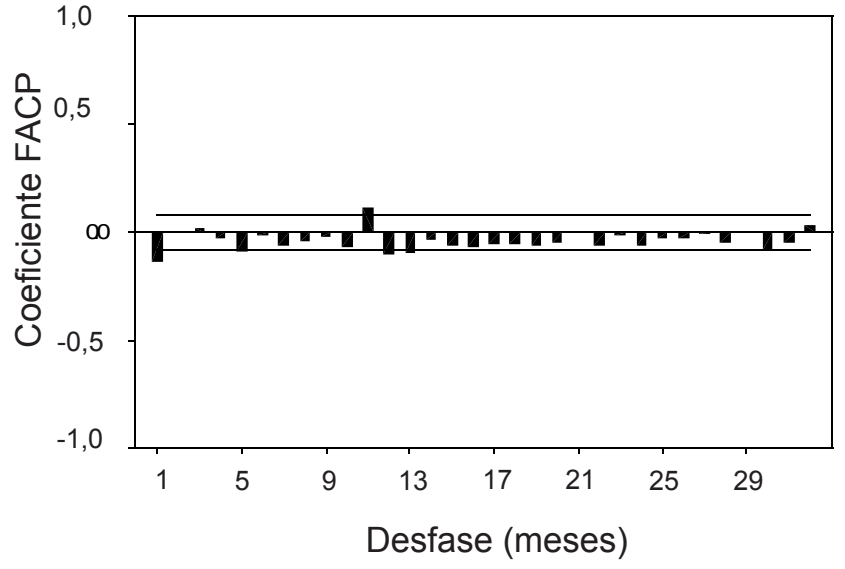

Figura 9. FACP estimada de la serie transformada con logaritmo y diferenciada con desfase $(\mathrm{d}=1)$.

1982-83, 1986-87 y 1997 con modelos de diferentes órdenes para cada año, los cuales lograron simular los episodios cálidos (Fig. 12), a excepción de El Niño 1982-83 y El Niño 1997-1998, que no alcanzó la intensidad observada, pero si logró prever condiciones cálidas durante este evento. Estas discrepancias se deberían a que el ENOS presenta procesos no lineales tales como: el intercambio de calor en superficie entre la atmósfera y el océano, así como, entre el océano y la tierra en la línea de costa; y por lo tanto el uso de un modelo lineal como el ARIMA, es limitado. A su vez, estos períodos presentan un gran ruido debido a la alta variabilidad atmosférica, la cual se suma a la variabilidad oceánica en los modelos univariados como el ARIMA. En cambio, en los modelos numéricos que tienen un forzante atmosférico separado de la dinámica interna oceánica, éstos dependen menos del ruido y más de las condiciones iniciales (Chen et al. 2004).

Para la validación cuantitativa se usaron los mejores modelos obtenidos (modelo 7 y modelo 8), y se simuló el Índice de Oscilación Peruano (IOP) durante el periodo julio 2004 al abril 2005. La validación de las previsiones se realizó usando los errores de predicción, y de manera resumida mediante la desviación media absoluta (DAM), la raíz cuadrada del error medio cuadrado (REMC) y el porcentaje de error medio absoluto (PEMA). Los estadísticos de error indicaron que el modelo 8 tuvo menor error de predicción para un horizonte de 10 meses (Tabla 4).

Para la validación cualitativa, se usaron los terciles del promedio trimestral para establecer las condiciones mensuales (fría, neutra y cálida) del IOP observado y del simulado con el modelo 8 (Tabla 5). Las simulaciones del IOP mediante el modelo 8

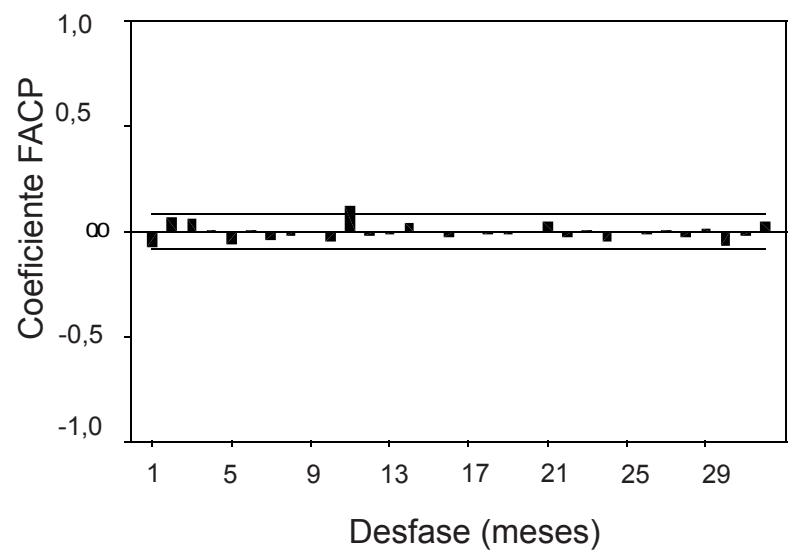

Figura 11. FACP estimada de los residuos del Modelo 1. 


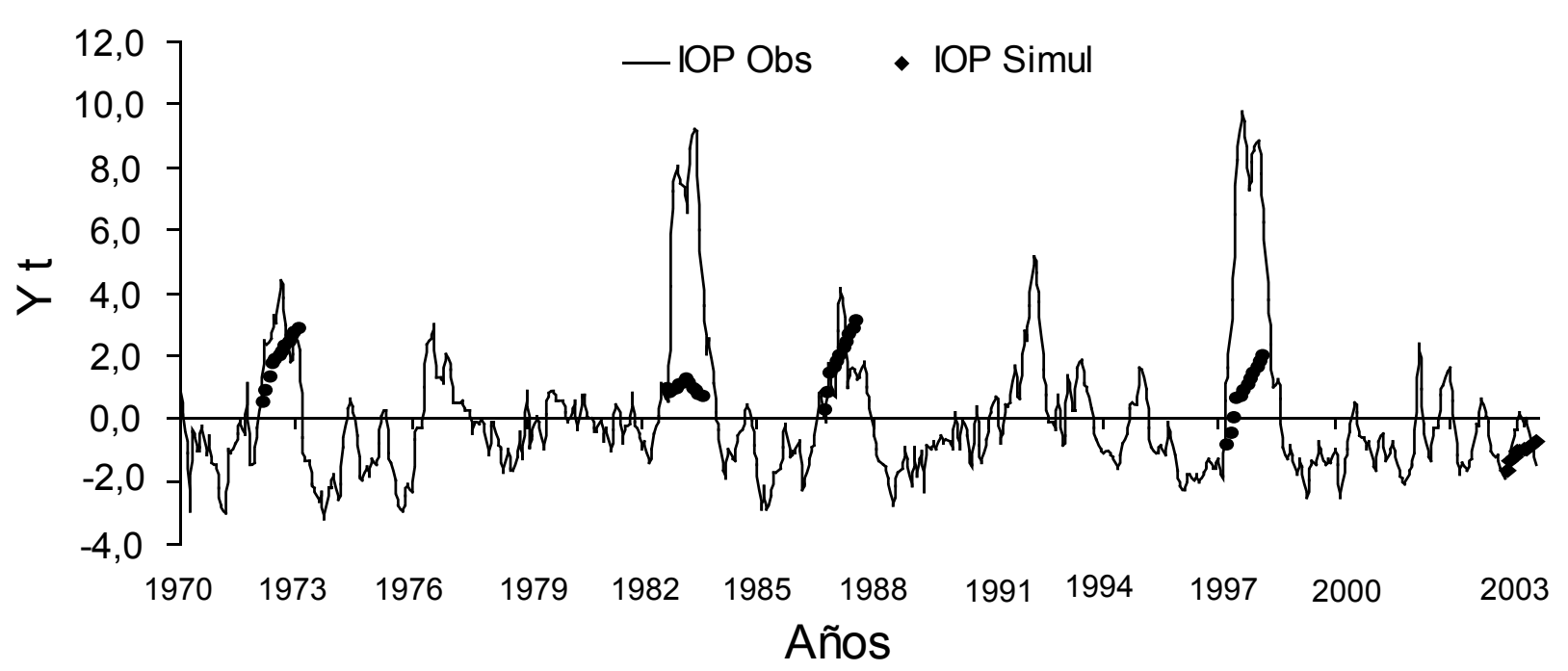

Figura 12. Variación temporal del Índice Oscilación Peruano (IOP) simulado, durante los años 1972, 1982-83, 1986-87, 1997, 2004-05.

Tabla 4. Validación cuantitativa de los Modelos 7 y 8 usando criterios de error de previsión para el periodo Julio 2004 a Abril 2005.

\begin{tabular}{ccc}
\hline & Modelo 7 & Modelo 8 \\
\hline REMC & 1,276 & $\mathbf{0 , 7 0 1}$ \\
DAM & 1,145 & $\mathbf{0 , 5 7 9}$ \\
PEMA & 16,273 & $\mathbf{8 , 5 6 7}$ \\
\hline
\end{tabular}

Tabla 5. Validación cualitativa del Modelo 8 para el periodo Julio 2004 al 2005.

\begin{tabular}{ccc}
\hline Fecha & $\begin{array}{c}\text { Condición simu- } \\
\text { lada }\end{array}$ & $\begin{array}{c}\text { Condición ob- } \\
\text { servada }\end{array}$ \\
\hline Jul-04 & Fría & Fría \\
Ago-04 & Fría & Fría \\
Sep-04 & Fría & Fría \\
Oct-04 & Fría & Neutra \\
Nov-04 & Fría & Neutra \\
Dic-04 & Fría & Neutra \\
Ene-05 & Neutra & Neutra \\
Feb-05 & Neutra & Neutra \\
Mar-05 & Neutra & Neutra \\
Abr-05 & Neutra & Fría \\
\hline
\end{tabular}

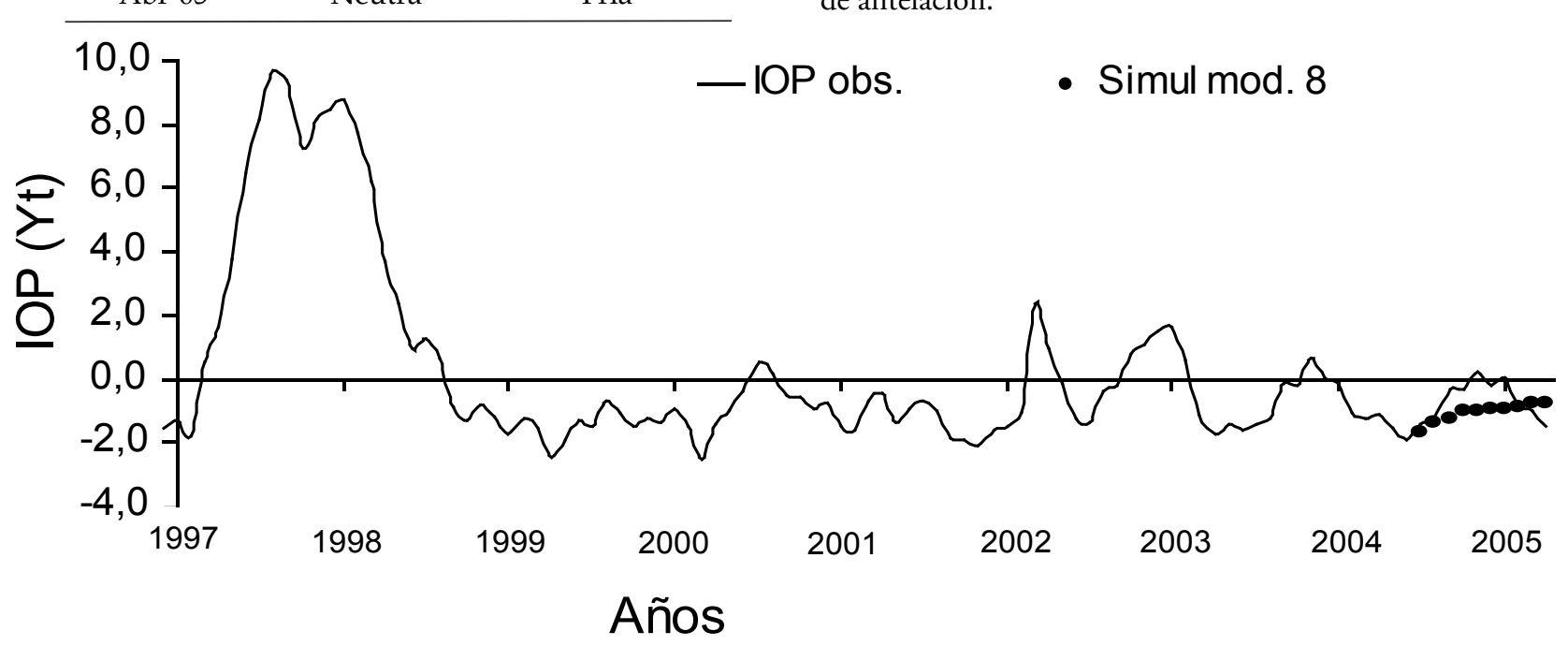

Figura 13. Previsión del Índice de Oscilación Peruano (IOP) mediante el Modelo 8. indicaron en promedio condiciones mensuales frías entre julio y diciembre del 2004 y condiciones neutrales a inicios del 2005. En este horizonte, se espero que el valor del IOP simulado sea mínimo en julio 2004 y máximo en abril 2005 (Fig. 13).

La validación cualitativa resumió de manera sencilla la concordancia entre los valores simulados y observados del IOP, mientras que la validación cuantitativa podría presentar valores altos de las medidas de errores (DAM, REMC, PEMA) debido a la variabilidad de las series.

La aplicación de este modelo permitió prever de una manera simple, el efecto de El Niño frente a la costa peruana.

\section{Conclusiones y recomendaciones}

El modelo autorregresivo y de medias móviles permitió prever a corto plazo los efectos El Niño frente a la costa peruana. Las previsiones según el modelo ARIMA $(12,0,11)$ indicó condiciones mensuales frías entre julio y diciembre del 2004 y condiciones neutrales a inicios del 2005, lo cual coincidió con las condiciones mensuales observadas.

El mejor modelo, indicó que la variabilidad de las temperaturas depende con mayor peso del valor de temperatura del mes anterior, y con menor peso de procesos registrados con un año de antelación. 
El modelo es lineal, univariado y dependiente de procesos costeros, lo que limita su horizonte de previsión. Una alternativa es usar modelos multivariados que tomen en cuenta variables en mesoescala y/o macroescala del Océano Pacífico.

\section{Agradecimientos}

Los autores agradecemos a la Dirección de Investigaciones Oceanográficas del IMARPE por proporcionar los datos de los sus laboratorios costeros. Al proyecto CENSOR (Climate variability and El Niño Southern Oscillation: Impacts for natural Resources and Management). Así mismo, se reconoce el apoyo de todo el personal del CIMOBP (IMARPE), en especial al Dr. Jorge Tam por sus valiosos comentarios acerca de este trabajo.

\section{Literatura citada}

Box, G., G. Jenkins and G. Reinsel. 1994. Times Series Analysis: Forecasting and Control. 3ra ed. Prentice Hall, Englewood Cliffs.

Chatfield, C. 1996. The Analysis of Times Series: A Introduction. 5 ta ed. Chapman \& Hall.

Chen, D., M. A. Cane, A. Kaplan, S. E. Zebiak y D. Huang. 2004. Predictability of El Nino in the past 148 years. Nature, 428, 733-736.

Enfield, D., E. Alfaro y E. Soley. 2003. Principios de Pronósticos Climáticos Estadísticos. NOAA Oficina de Programas Globales. Tercer Foro de Perspectiva Climática para la Costa Oeste de América del Sur (COF3-WCSA).
Goodrich, R. 1989. Applied Statistical Forecasting, Belmont, MA: Business Forecast System, Inc.

McPhaden, M.J. 2001. El Niño and La Niña: Causes and Global Consequences. Nacional Oceanic and Atmospheric Administration, John Wiley \& Songs Inc. Seattle, WA,USA.

NOAA Magazine. 2003. NOAA gets U.S. consensus for El Niño/La Niña index, definitions. Sept. 30, http://www.noaanews. noaa.gov/stories/s2095.htm

Purca, S., Antezana, T. and Riquelme, R. 2005. MS. The peruvian oscillation index. Capitulo 2. en Purca, S. Variabilidad temporal de baja frecuencia en el Ecosistema de la Corriente Humboldt frente a Perú. Tesis de Doctorado. Universidad de Concepción, Chile.

Purca, S. 2005. Variabilidad temporal de baja frecuencia en el Ecosistema de la Corriente Humboldt frente a Perú. Tesis de Doctorado. Universidad de Concepción, Chile.

Uriel, E. and Peiró, A. 2000. Introducción al análisis de Series Temporales, Editorial AC.

Woodman, R. 2005. Modelo Estadístico de Pronóstico de las Precipitaciones en la Costa Norte del Perú. Sociedad Geológica del Perú, volumen especial $\mathrm{N}^{\circ} 6$ en homenaje al Ing. Alberto Giesecke, p. 25-34. 
